\title{
TARIFFE, DAZI E POLITICHE DI COMMERCIO
}

\author{
ANGELO MOIOLI (*)
}

RIASSUNTO. - Nella storia delle riforme civili in campo economico che l'Illuminismo lombardo ha fatto proprie, la questione dei regimi doganali e delle politiche commerciali gode da tempo una centralità del tutto speciale. A renderla tale è la consapevolezza di come essa abbia assunto uno specifico significato modernizzante, anche se riferito solo a una parte della regione insubrica, quella sottoposta al dominio degli Austrias, vale a dire la Lombardia asburgica. Questa presentava un sistema di regolazione daziaria dell'interscambio del tutto particolare. La sua difformità consisteva nel fatto che nel territorio dello Stato di Milano vigevano dei dazi interni come espressione delle cinque entità sovrane in cui esso si scomponeva. Ciò voleva dire che le singole province trattavano le merci giunte da altri distretti dello stato non come articoli provenienti dal suo interno, ma come se addirittura venissero introdotte da territori esteri. Ma era giunto anche il momento in cui il governo viennese aveva insediato una giunta, nel 1764, con l'obiettivo di modificare alla radice questo sistema. Tra i membri di giunta figurava anche il giovane patrizio milanese Pietro Verri, proprio in quanto autore di un testo rimasto lungamente manoscritto dal titolo "Considerazioni sul commercio dello Stato di Milano". La sua importanza ben apprezzata da Vienna derivava dal fatto che in esso veniva per la prima volta dimostrata la connessione tra misure tariffarie e bilancia di commercio. Attraverso questa connessione, la bilancia commerciale si apriva quindi a quella dei pagamenti e ciò avrebbe indotto Schumpeter a celebrare in Verri un autentico econometrico. Sarà stato anche così, ma in ogni caso le elaborazioni compiute in seguito da Verri insieme al Maraviglia Mantegazza per il 1762 non avevano portato ad alcun concreto risultato per quanto riguarda la riforma dei dazi cui si aspirava. Sarebbe tuttavia toccato di nuovo al Verri tentare la via della riforma daziaria, questa volta sulla base di una nuova bilancia commerciale portata a termine nell'agosto del 1773.

La sua proposta consisteva nel sostituire dei dazi unici di confine a quelli multipli cui continuava a riferirsi il sistema di imposizione in atto. Non avrebbe comunque avuto fortuna, perché la vera riforma, pur concepita secondo quei principi, sarebbe stata introdotta nel 1786.

${ }^{(*)}$ Istituto Lombardo Accademia di Scienze e Lettere; Università Cattolica, Milano. 
Il bilancio di commercio su cui questa si basava, avrebbe però allora avuto come riferimento il 1778 e sarebbe stata opera di un suo collaboratore per la verità non molto "illuminato", dal nome di Baldassarre Scorza.

ABSTRACT. - Tariffs, duties and commercial policy.

Duty systems and trade policies were central in the reforms proposed by the Lombard Enlightenment economists for the State of Milan. This State had originally adopted a duty scheme characterized by the presence of internal duties originating from each one of the five sub regional entities, i.e. the provinces of the State. That means that the single provinces treated the commodities coming from other provinces of the State not as home items but as if they were commodities coming from abroad. In 1764 the Vienna government established a special commission with a view to change the system. The Commission included a young Milanese patrician, Pietro Verri, the author of Considerazioni sul commercio dello Stato di Milano (Reflections on trade in the State of Milan). The treatise was highly appreciated in Vienna since it was the first to show the relation between the tariffs and the trade balance or the balance of payments. This explains why Schumpeter credited Verri as an early econometrician. Nevertheless, the studies carried out by Verri (together with Maraviglia Mantegazza) in 1762 did not lead to any practical reform of the duty system. Similarly, a new proposal suggesting to substitute a single border toll for the whole host of internal duties, did not produce any practical result. The proposal was based on a new study of the balance of trade completed in August 1773. Finally, Verri had no role in the reform adopted later in 1786, even if that was conceived on the basis of principles he had himself stated in his second attempt. But the balance of trade from which the reform was designed had been produced by Baldassarre Scorza, who was rated inferior to Verri as an 'enlightened' political economist.

Nel riconsiderare il percorso storico lungo il quale ha preso forma e consistenza in Lombardia il disegno riformatore che durante la seconda metà del Settecento l'istanza illuministica dell'incivilimento ha concorso a concepire e a realizzare sul piano economico, balza evidente quale rilevanza abbia assunto la questione del commercio come strumento di politica economica. Così almeno è stato soprattutto nel caso dello Stato di Milano, dove infatti la tematica della regolazione dei flussi di merci sia all'interno che con l'estero è stata affrontata in una prospettiva di revisione più radicale.

$\mathrm{Ha}$ avuto in questo la sua parte il fatto che in tale contesto e proprio a partire dagli anni '60 del secolo, ha operato quella che Schumpeter avrebbe poi invitato a riconoscere come la "scuola di 
Milano" dell'economia politica pre-smithiana ${ }^{1}$ e che raccolta intorno al periodico "Il Caffè", "sotto l'orgogliosa insegna” dell'Accademia dei Pugni, ${ }^{2}$ si richiamava alla concezione dell' "economia civile" teorizzata da Antonio Genovesi, reinterpretandola come scienza di governo e sulla base di un accostamento condotto per via empirica. Ciò era reso tra l'altro possibile dall'essere alcuni suoi esponenti dei pubblici funzionari, da posizioni certamente di primo piano nell'amministrazione della Lombardia Austriaca e proprio per questo in grado di agire, secondo quanto auspicava il Kaunitz, non come avrebbe fatto un "filosofo da gabinetto" e bensì un "filosofo pratico" votato "all'esperimento politico in materia di pubblica economia".

$\grave{E}$ quanto poi si è verificato quando in sede governativa si sono affrontati i nodi per un verso della politica doganale e per l'altro della libera circolazione interna delle merci, a cominciare da quella dei grani. Sul primo fronte, quello del regime daziario, si imponeva una revisione di particolare ampiezza perché nella parte asburgica della regione il combinarsi dei dazi interni con quelli di confine era tale da gravare più sulle correnti mercantili infrastatali, che non su quelle da e per l'estero. Lo stesso non avveniva nelle aree politiche adiacenti, uscite dalla scomposizione della Lombardia viscontea, per lo meno con una valenza così discriminante. Solo nello Stato milanese in effetti vigeva un sistema di oneri e di vincoli imposti a livello infrastatale secondo una articolazione dello stesso in cinque entità sovrane, che si confrontavano tra loro in modo da rendere più onerosi i passaggi di beni dall'una all'altra e da favorire per contro gli scambi di ciascuna di esse con l'estero. Avveniva così che ognuna di tali sezioni daziarie trattasse le merci giunte dalle altre provincie dello Stato non come articoli di quella provenienza, ma addirittura come se fossero fatte circolare da e per territori posti oltre confine. Ecco perché continuavano a mantenersi i così detti "dazi di traverso" che colpivano la circolazione interna pur in misura assai dif-

1 P.L. Porta, Lombard Enlightenment and Classical Political Economy, in «The European Journal of the History of Economic Thought», London, a. XVIII (2011), p. 522. 2002, p. 189

C. Capra, I progressi della ragione. Vita di Pietro Verri, Bologna, Il Mulino,

A. Carera, Cesare Beccaria magistrato in "provincia d'Annona", in AA.VV., Cesare Beccaria tra Milano e l'Europa. Convegno di studi per il $250^{\circ}$ anniversario della nascita, Milano, Cariplo-Laterza, 1990, p. 443. 
ferenziata, a seconda dei percorsi seguiti e della convenienza anche sotto il profilo dei costi della distanza. Non si riusciva in effetti a superare la dicotomia tra l'unità del territorio statale e la sfera di sovranità propria delle sue provincie daziarie.

Non sfuggiva del resto agli organi di governo dei Paesi confinanti questo modo anomalo di procedere, anche perché durava ormai da tanto tempo. Va fatto valere al riguardo, anche in questa sede, quanto si annotava proprio negli anni '60 del Settecento in una relazione anonima inoltrata ai Cinque Savi della Mercanzia a Venezia: "La legge principalmente delle Finanze ci ha fatto sempre conoscere questo disordine nello Stato di Milano, mentre con sorpresa de' politici s'è osservato che la Contessa di Como sosteneva una guerra formale con la Principessa di Pavia, e questa con la Contessa di Lodi, e la Contessa di Lodi con quella di Cremona, e tutte insieme contro la Duchessa di Milano, la quale dal canto suo la sosteneva con tutte le altre".${ }^{4}$ C'era un po' di sarcasmo in questa colorita denuncia del sistema in atto. Forse anche perché si trascurava di riconoscere che la persistenza delle giurisdizioni daziarie interne aveva una sua logica. Consentiva se non altro di assicurare al pubblico erario dei proventi che altrimenti avrebbero dovuto essere recuperati attraverso l'inasprimento dei diritti di confine, con la conseguenza però di ripercuotersi negativamente sui legami di complementarietà intrattenuti dalle singole articolazioni territoriali appena rammentate con le limitrofe aree di altri Stati.

Tuttavia se anche si fosse voluto derogare dal regime doganale ereditato da un lontano passato, il superamento della sua impostazione a dazi multipli si sarebbe rivelato problematico per almeno un'altra ragione. Fintanto che l'applicazione del relativo congegno tariffario fosse stato sottoposto al controllo di appaltatori, non si sarebbero potute introdurre modifiche sostanziali nei trattamenti daziari concordati con loro. E non è detto che una volta giunti alla scadenza del contratto (o dei contratti) di locazione in corso, l'adozione di una gestione in economia del "dato della mercanzia" si traducesse in un cambiamento della sua impostazione, soprattutto se la prospettiva era di tornare prima o poi a praticare la via dell'affitto. Così nel 1725 , dopo che

4 A. Moioli, Pietro Verri e la questione della riforma daziaria nello Stato di Milano, in C. Capra (a cura di), Pietro Verri e il suo tempo, Milano, Il Cisalpino, 1999, t. II, p. 853. 
durante il governatorato del Colloredo era stato giocoforza assumere direttamente in sede governativa la riscossione dei tributi daziari, si era bensì riusciti ad affrontare un riordino dei tariffari vigenti, ma pur sempre avendo di mira una loro riclassificazione condotta nella piena conferma dei criteri ordinatori del sistema di imposizione preesistenti. ${ }^{5} \mathrm{E}$ quell'assetto tariffario era rimasto tale anche quando nel 1751 si era avviata la Ferma Generale dei tributi indiretti, proprio a cominciare da quelli delle dogane.

Per la verità si sarebbe anche voluto reimpostarne la riscossione su nuove basi, ma la giunta posta in essere allo scopo nel 1755 dal plenipotenziario Cristiani si era rivelata "un corpo senz'anima" che nel giro di nove anni "non era venuta a capo di nulla" ${ }^{6}$ E tutto era continuato come prima, almeno fino al 1765 , l'anno in cui era giunto a scadenza l'appalto generale delle gabelle, dopo una prima riconferma nel 1757 in capo ai medesimi titolari.

$\mathrm{Ma}$ a quel punto si era ormai raggiunta in sede governativa, a Vienna come a Milano, la piena consapevolezza che occorresse una decisa correzione di marcia al riguardo. Il ricorso alla locazione del così detto "Dato della Mercanzia" non era per questo messo in discussione e tuttavia ora lo si faceva valere in cointeressenza con il regio fisco, che doveva così garantire a quest'ultimo una quota di proventi non più limitata soltanto al canone di sua spettanza. L'attenzione sembrava invece focalizzarsi ora sulla reimpostazione quali-quantitativa del gettito, affinché lo si potesse associare a una manovra razionalizzatrice dei flussi commerciali da tassare.

Sarebbe stata però allora una nuova giunta "interinale" a farsene carico, se non attraverso una rifusione complessiva delle tariffe, per via di una loro mirata rettifica in alcune direzioni. Un compito questo comunque cui Kaunitz attribuiva grande importanza, assertore come era divenuto nel frattempo - lo è stato fatto notare giustamente - del valore strategico assunto dalla "tariff reform" ai fini della rivitalizzazione del commercio e dell'industria. ${ }^{7}$ E si può perciò anche capire la sua insistenza nel sostenere la necessità che il nuovo organismo fosse formato da "soggetti spregiudicati, intesi delle buone teorie commerciali e consumati di quel

\footnotetext{
5 Ivi, pp. 860-861.

6 Ivi, p. 870.

7 Ivi, p. 869.
} 
mercimonio". Gli sarebbero però di li a poco andate anche bene "persone di nascita, di educazione e di buoni principi nelle cose commerciali", tra le quali accettava di annoverare pure "due giovani cavalieri, figli di ottimi genitori ed applicatissimi agli studi" come Angelo Maria Maraviglia Mantegazza e Pietro Verri. E se nei confronti del primo più che le qualità personali avevano contato la condizione patrizia della famiglia e i ruoli certamente di prestigio ricoperti dal padre nelle cariche pubbliche, per il secondo i motivi della cooptazione non erano però stati soltanto quelli. Anzi, le difficoltà da lui vissute e mai venute meno nei rapporti familiari non lo avevano aiutato molto. Aveva però anche altre carte da giocare. Stava diventando proprio allora il principale animatore della già accennata "scuola di Milano", della corrente di pensiero quindi che faceva della disciplina economica "un pilastro fondamentale dell'idea dell'illuminismo civile", come è stato ben detto. ${ }^{8}$ Tanto più che il suo attivismo a tale titolo gli stava procurando già una certa notorietà al di fuori dell'ambiente ambrosiano, non senza suscitare attenzione anche a Vienna, almeno nella ristretta cerchia di funzionari che facevano capo al Dipartimento d'Italia. Lui del resto non aveva trascurato di farsi conoscere anche altrimenti, onorando una consuetudine allora in auge presso gli aspiranti a pubblici uffici di un certo rango e quindi affidando a un proprio elaborato appositamente redatto la dimostrazione delle abilità di ordine propositivo e operativo richieste per assumere un determinato incarico.

Il 1763 era stato appunto l'anno in cui egli aveva trovato il modo di far pervenire direttamente al Kaunitz in versione manoscritta una memoria dal titolo piuttosto incolore, ma dai contenuti ben diversamente impegnativi e tutt'altro che di circostanza, come le "Considerazioni sul commercio dello Stato di Milano". Se ne aveva ricavato delle ottime credenziali, era stato certamente per l'originalità della trattazione, ma ancor più per la forza argomentativa del relativo impianto.

Soprattutto ad imporlo all'attenzione delle autorità di governo viennesi e ad assicurargli una investitura senza riserve in qualità di consigliere della nuova "daziaria", era l'essersi rivelato in piena sintonia con gli orientamenti maturati nel frattempo presso la Cancelleria pro-

8 P.L. Porta, Nuove prospettive negli studi economici sull'illuminismo lombardo. Relazione conclusiva del convegno, infra. 
prio in materia doganale. Si leggeva infatti in quelle sue pagine: "Le Tariffe (----) sono il primo mobile per la direzione del commercio: sono elleno (---) la parte più preziosa dell'economia politica, ed il capo d'opera della legislazione, poiché dalle Tariffe dipende in gran parte il rendere il commercio d'una Nazione utile o rovinoso". 'Del resto al Kaunitz e ai suoi collaboratori non poteva sfuggire l'efficacia con cui Verri aveva fatto proprio un approccio storico-empirico alle questioni trattate. Era lui stesso ad avvertire che nella prima parte del suo scritto dedicata alle "Cagioni della grandezza e decadenza del commercio di Milano", aveva inteso ricostruire una "storia economica" della propria città e del suo Ducato tra Quattro e Settecento. ${ }^{10}$ Ciò non poteva non trovare d'accordo lo stesso Kaunitz, se ai primi del 1764 si era sentito in dovere di far notare alla sua imperatrice e proprio con riferimento alla politica daziaria da riformare come "né meno le teorie per giuste che siano e le idee astratte ancorché in se stesse bellissime, a nulla vagliono quando non si sappiano applicare alle varie circostanze del luogo" e delle quali "non può acquistarsi la scienza né cogli sforzi d'ingegno, né colla lettura" e bensì in stretto contatto con la realtà fattuale. ${ }^{11}$ Anche per lui quindi era da privilegiare quel tipo di accostamento alla economia politica che abbiamo poi imparato a qualificare come il prodotto di una cultura illuminista, ben sapendo che esso sarebbe poi stato messo da parte con l'imporsi del metodo logico-deduttivo. ${ }^{12}$

Sempre muovendosi entro queste coordinate, Verri aveva mostrato però di saper spendere la credibilità della propria dissertazione anche su un altro piano. Egli aveva infatti messo al centro della seconda parte della stessa, la connessione tra la manovra delle dogane e la misurazione in quantità e in valore dell'interscambio. La costruzione della bilancia commerciale diventava così per lui un presupposto di metodo irrinunciabile ai fini di qualsiasi ipotesi di intervento sul com-

9 P. Verri, Stato attuale del commercio di Milano, in Edizione Nazionale delle Opere di Pietro Verri, vol. II, Scritti di economia, finanza e amministrazione. A cura di G. Bognetti, A. Moioli, P.L. Porta, G. Tonelli, Roma, Edizioni di Storia e Letteratura, 2006, t. I, p. 247.

10 A. Moioli, Nota introduttiva, in idem, p. 24.

11 Idem, La questione della riforma daziaria nello Stato di Milano, cit., p. 870.

12 C. Perrotta, Il contributo di Genovesi alla teoria illuminista dello sviluppo, in A. Roncaglia (a cura di), Moneta e sviluppo negli economisti napoletani dei secoli XVIIXVIII, Bologna, Il Mulino, 1995, vol. I, pp. 73-75. 
plesso tariffario del "dato della mercanzia". Restava per altro questa la via obbligata anche per poter decidere se continuare o meno a praticare la formula dell'appalto generale per l'esazione delle gabelle. Se si era contro una sua riproposizione - e Verri lo dichiarava apertamente in nome dei pesanti limiti che essa comportava - bisognava comunque farlo sulla base di una adeguata conoscenza dei movimenti dei flussi mercantili che solo una stima degli stessi in entrata e in uscita era in grado di assicurare.

L'estensore di quella memoria aveva dunque colto nel segno quando aveva deciso di inserire nel testo la statistica dell'import/export per il 1752, che sappiamo rifarsi alla rilevazione ordinata per tale anno dal ministro plenipotenziario Cristiani e poi rimasta sino ad allora inutilizzata, oltre che per certi aspetti ancora da completare. Il suo era quindi un elaborato derivato da una fonte rimasta di necessità ufficiosa e che solo lui però si era posto a valorizzare, nella consapevolezza di compiere un atto di per sé innovativo, il cui significato tra l'altro, da un punto di vista metodologico, andava ben al di là di quello che si sarebbe potuto assegnare ai conteggi effettuati in materia e nello stesso periodo da altri "bilancisti" ambrosiani (così li avrebbe chiamati l'Einaudi) come il Carpani e il Muttoni. ${ }^{13}$

A ragion veduta perciò egli, già durante la prima riunione di giunta tenutasi il 21 febbraio 1764, si era sentito in dovere di affermare: "non potersi aggravare o solevare una merce nella nuova tariffa senza $\mathrm{i}$ lumi che dovevano scaturire dal Bilancio del nostro Commercio" e neanche "salvare i diritti della regalia coll'accrescere o sminuire il tributo di una merce senza conoscerne l'annuo prodotto". ${ }^{14}$ Solo che ben sapeva che le sue rischiavano di apparire puramente delle dichiarazioni di principio. Sarebbe stato diversamente se gli fosse riuscito in quella sede di utilizzare i dati della stima di bilancio da lui compiuta per il 1752. Bisognava però allora estrapolarli dal testo inoltrato a Vienna e a ciò Verri non era stato certo autorizzato. Tanto più che per essi valeva la regola della riservatezza, cui egli non poteva certo sottrarsi. In alternativa non restava che tentare l'azzardo, dando alle stampe quel prospetto con le relative glosse che l'accompagnavano, seppure in un

13 A. Moioli, Nota introduttiva, in Edizione Nazionale delle Opere di Pietro Verri, vol. II, t. I, p. 466.

14 Idem, La questione della riforma daziaria nello Stato di Milano, cit., p. 872. 
numero limitato di copie. Nel farlo egli si era anche altrimenti esposto perché la versione pubblicata, pur dopo le rettifiche recate a quella manoscritta, presentava un disavanzo così ingente da metterne in dubbio la credibilità.

Per la verità Verri aveva tentato di rendere quest'ultimo meno incongruo, inserendo nel relativo conteggio anche delle partite invisibili e prima fra tutte quella dei trasferimenti connessi con gli investimenti fondiari e finanziari compiuti all'estero, a cominciare dalle rendite che i proprietari milanesi e pavesi ricavavano dai loro possessi nei territori al di là del Ticino ceduti al Regno Sardo-Piemontese e fatte apprezzare attraverso una incognita, secondo una relazione inversa che rifletteva un rapporto proporzionale del loro ammontare rispetto a quello del saldo passivo di bilancio. ${ }^{15}$

Del resto le aspre critiche rivoltegli in quella occasione non avevano riguardato né il procedimento di calcolo dei saldi, né i risultati che se ne erano ricavati. Solo il questore Pellegrini che tanta parte stava avendo nei lavori della giunta, gli aveva avanzato delle obiezioni di merito, a cominciare da quella che metteva in discussione la possibilità di derivare dalla stima presentata un criterio uniforme di revisione complessiva della tariffa, come invece il suo estensore avrebbe voluto. Gli sembrava per altro opinabile la scelta di un anno di riferimento come il 1752 nel quale, ormai chiusa l'ultima parentesi bellica di quel tormentato periodo, le importazioni avevano subito una impennata anomala, ripercuotendosi pesantemente sull'entità del saldo passivo. Non era poi neppure fuori luogo il suo appunto circa il fatto di aver trascurato la dimensione della circolazione mercantile interna rispetto a quella da e per l'estero, pur sapendo quanto fosse penalizzata nel contesto del vigente regime a dazi multipli. ${ }^{16}$

In quanto al Kaunitz che pur aveva così duramente ripreso il Verri per aver dato pubblicità a quel bilancio da tenere invece riservato, si era ben guardato dallo sminuirne il significato, come sarebbe stato se avesse avallato le stime che sempre allo stesso titolo e con esiti per lo meno più tranquillizzanti, andavano offrendo proprio il Carpani e il Muttoni già ricordati. Anzi, visto che proprio con quell'elaborato "si era rotto il

15 Idem, Nota introduttiva, in Edizione Nazionale delle Opere di Pietro Verri, vol. II, t. I, p. 463.

16 Idem, La questione della riforma daziaria nello Stato di Milano, cit., p. 872. 
ghiaccio", gli era parso opportuno trarne motivo per affrontare su nuove basi e questa volta con tutti i crismi della ufficialità, un ricalcolo analogo, che si sarebbe allora riferito al $1762 .{ }^{17}$

Su chi, all'interno della giunta, avrebbe dovuto occuparsene, non vi erano stati dubbi. L'unico che aveva mostrato di sapercela fare per avere acquisito di sua iniziativa le tecnicità necessarie, era pur sempre l'autore dell'elaborato precedente, anche se coadiuvato ora dal Meraviglia Mantegazza. Sotto la sua guida e attraverso un poderoso sforzo organizzativo e finanziario, l'opera era stata portata a termine con successo entro l'ottobre del 1765 . Si era trattato, a suo dire, di un vero e proprio "monumento" da celebrare nella certezza che "in nessun altro tempo e Stato siasi fatta una simile operazione, giustificata come questa" ${ }^{18}$ Ben a ragione poteva affermarlo, essendosi rivelate infondate certe critiche che in tempi più ravvicinati sarebbero state rivolte dall'Einaudi alla struttura per soli saldi di quel conto. ${ }^{19}$ Del resto, lo Schumpeter non aveva avuto dubbi al riguardo. Per lui Verri era da ricordare come "one of the first economists to figure out a balance of payments" che con le stime predisposte allo scopo aveva mostrato di sapere "how weave fact-finding and theory into a coherent tissue". ${ }^{20}$

Se lo si poteva definire così, era pur sempre a motivo della visione strategica entro la quale egli collocava la statistica del commercio con l'estero. Si può anche convenire che egli vi si accostasse alla ricerca di un suo equilibrio dinamico, "come tale proiettato verso la crescita sincronica delle componenti attive e passive" ${ }^{21}$ Ma proprio perché era dentro a questa logica, egli vi ravvisava la fonte dei "lumi" intorno ai quali doveva imbastirsi la riforma tariffaria da affrontare, che ne restava pur sempre il principale obiettivo. A maggior ragione così doveva avvenire se si voleva mettere fine a un regime impositivo che con la sua intelaiatura a giurisdizioni separate, veniva ad ostacolare i traffici con gli Stati terzi poggianti sullo scambio di merci contro merci. E pro-

17 Ivi, p. 878

18 Idem, Nota introduttiva, in Edizione Nazionale delle Opere di Pietro Verri, vol. II, t. I, p. 469.

19 Ivi, pp. 473-474.

20 J.A. Schumpeter, History of Economic Analysis edited from manuscript by Elisabeth Boody Schumpeter, London, George Allen \& Unwin, 1954, p. 178.

21 G. Bognetti, A. Moioli, P.L. Porta, Illuminismo lombardo ed economia politica classica. Una replica, in «Il pensiero economico italiano», a. XVI (2008), n. 2, p. 221 
prio perché allora era convinto che questi dovevano essere comunque favoriti rispetto a quelli che comportavano solo esborsi di denaro (in omaggio quindi a reminiscenze mercantilistiche non ancora sopite), ${ }^{22}$ non gli era rimasto che chiedere con forza, fin dalle prime sedute di giunta, la rimozione di ogni impedimento a tale riguardo, quale altrimenti il persistente sistema delle unità doganali multiple era in grado di perpetuare. Non restava però allora che abolire le gabelle intermedie connesse, a tutto favore di una loro rifusione in un gettito alimentato soltanto da dazi di confine uniformi cui sottoporre le merci da qualunque parte dello Stato entrassero o uscissero. Ma gli sarebbe stato da subito anche chiaro che finalizzando in quel modo la costruzione del bilancio di commercio, la concezione illuministica sulla quale si sorreggeva rischiava di non poter giungere a uno sbocco concreto. In effetti proprio mentre prendeva corpo la rilevazione per il 1762, era divenuto evidente come questa sarebbe proceduta ormai "segregata dal punto delle tariffe". Lo annunciava con senso di soddisfazione il fermiere Mellerio al suo sodale Greppi, ${ }^{23}$ ben consapevole che così agendo, il questore Pellegrini incaricato di ricomporre il quadro daziario, vi avrebbe fatto fronte non certo con propositi di riforma radicale e bensì soltanto nella prospettiva di apportare delle rettifiche parziali al tessuto impositivo ereditato dal vecchio sistema.

Per Verri questa non era altro che la via "del rappezzo giornaliero", praticando la quale, la neutralizzazione funzionale di quel bilancio era diventata inevitabile a tal punto da renderlo "roba seppellita e sinora inutile". Non poteva per altro rammaricarsi più di tanto perché quando nel 1768 si esprimeva in questi termini ${ }^{24}$ aveva già avuto modo di verificare, in qualità di delegato regio nella Ferma mista fin dal 1766, come il Pellegrini che pur gli si era contrapposto, avesse compiuto tutto sommato un buon lavoro, realizzando una prima efficace razionalizzazione dei carichi daziari preesistenti. ${ }^{25}$ Tra i meriti che gli doveva riconoscere vi era senz'altro quello del trattamento di favore riservato alle importazioni di beni di lusso, la cui conferma gli appariva in linea con

22 G. Bognetti, Nota introduttiva, in Edizione Nazionale delle Opere di Pietro Verri, vol. II, t. I, p. 669.

23 A. Moioli, Nota introduttiva, ivi, p. 468.

24 Idem, La questione della riforma daziaria nello Stato di Milano, cit., p. 881.

25 Ivi, pp. 879-880. 
quanto lui stesso era venuto sostenendo fin dal 1763 circa l'opportunità di assicurare la libera circolazione nello Stato di tali generi come occasione per perpetuare al riguardo il ruolo centrale di intermediazione svolto da Milano nei confronti degli Stati confinanti. ${ }^{26}$

Del resto, contrariamente a quanto lui lasciava intendere, il suo progetto di rifusione complessiva delle dogane non era stato affatto accantonato e sarebbe infatti tornato alla ribalta di lì a qualche tempo, quando la fine anticipata della Ferma mista nel 1770 e la redenzione delle regalie alienate avviatasi nel frattempo, ne avevano fatto riscoprire l'attualità, incominciando da quella indicazione di metodo che ne era il presupposto, vale a dire il suo ancorarsi a una rigorosa misurazione dell'interscambio. E nuovamente Verri si era così trovato a guidare la compilazione di un altro bilancio di commercio, questa volta riferito al 1769 , mettendo per questo a frutto abilità di ordine logico-matematico e statistico di primo ordine, quali si attenderebbe fossero proprie della figura di econometrico evocata da Schumpeter, ${ }^{27}$ o se si vuole, secondo una terminologia più dimessa, di quella dell'aritmetico politico posta in campo di recente. ${ }^{28}$

Non deve perciò stupire la celerità con cui la rilevazione di una massa così ingente di dati è proceduta entro la metà del 1771, ma non meno il fatto che, chiamato il Verri ad altre incombenze altrettanto impegnative presso il nuovo Magistrato Camerale appena istituito, avesse lasciato dietro di sé un vuoto operativo rimasto a lungo incolmato. Sino a che, nella prima metà del 1773 un suo ulteriore intervento si sarebbe rivelato decisivo per rimettere in sesto su basi più rigorose e meno erronee le operazioni finali di stima nel frattempo compiute da altri. Ed era stato proprio allora che egli si era visto affidare per esplicita volontà di Kaunitz, il compito per il quale anche quel bilancio era stato concepito, vale a dire, adesso che il monitoraggio dell'interscambio diventava possibile in modo ancor più aggiornato, la progetta-

26 G. Tonelli, «Considerazioni sul lusso» nella riforma daziaria dello Stato di Milano (seconda metà del XVIII secolo), in A. Alimento, Modelli d'oltre confine, Prospettive economiche e sociali negli Antichi Stati Italiani, Roma, Edizioni di Storia e Letteratura, 2009, p. 281.

27 A. Schumpeter, History of Economic Analysis, cit., p. 178.

28 P.L. Porta, The "School of Milan". Competition and Public Happiness in Pietro Verri's Political Economy. Testo della Blanqui Lecture 2009 tenuta su invito della European Society for the History of Economic Thought, il 24 aprile 2009 a Tessalonica, p. 19. 
zione di una riforma veramente organica e non più quindi affidata a parziali rettifiche del vigente impianto tariffario. Il disegno da lui teorizzato fin dai suoi esordi nella giunta "interinale", sembrava dunque potersi finalmente realizzare, consentendogli per giunta di diventarne l'artefice principale.

Le sue idee in proposito non erano certo cambiate rispetto ad allora e anzi l'orientamento maturato a Vienna pareva, addirittura, rafforzarle. Il meccanismo delle giurisdizioni daziarie interne restava per lui il principale imputato, frutto di un "real scisma" tra le stesse che operava come "il vero tarlo del mercimonio milanese". ${ }^{29} \mathrm{E}$ se in precedenza egli lo aveva fatto dipendere dall'impossibilità di praticare un interscambio di merci contro merci, piuttosto che contro denaro, ora invece lo imputava ad un'assenza di libertà nel "mettere a concorrenza le Piazze forastiere", frutto di una "negoziazione spinta per quella parte per dove resta men gravoso il tributo" e come tale "sottoposta all'arbitrio di quella piazza a cui è sforzata di ricorrere". Con il risultato di provocare "alla fine maggior uscita di denaro dallo Stato di quello che sarebbe, qualora la differenza di prezzo non fosse largamente scontata dalla differenza del tributo". ${ }^{30}$

Ecco perché Verri metteva nuovamente al centro della sua proposta l'adozione di una tariffa unica ai confini dello Stato, previa l'eliminazione dei "dazi di traverso". Non poteva del resto avvertire un limite insito nel suo ragionamento e per il quale il fatto di considerare "tutto lo Stato di Milano come una unità" dal punto di vista doganale, non era di per sé la garanzia che ne sarebbero derivati effetti di crescita dell'interscambio, posto che, come la recente teoria economica ci ha insegnato, si stesse realizzando un'aggregazione tra porzioni territoriali caratterizzate da legami reciproci di scambio nettamente depotenziati rispetto a quelli intrattenuti con le realtà statuali confinanti. ${ }^{31}$ Egli era, peraltro, convinto che la circolazione interna delle merci sarebbe comunque aumentata di forza propria, come riflesso automatico dell'adozione di un regime daziario unificato. A tal punto da credere che quella componente non meritasse una specifica misurazione. Già in occasione del bilancio per il 1762

29 A. Moioli, La questione della riforma daziaria nello Stato di Milano, cit., p. 874.

30 Ivi, p. 883.

31 R.G. Lipsey, The Theory of Customs Unions: a General Survey, in «The Economic Journal», a. LXX (1960), pp. 496-513. 
si era dichiarato "che tutte le mercanzie che sarebbonsi trovate registrate (----) di semplice circolazione interna da una Provincia all'altra dello Stato medesimo, dovevano omettersi interamente, non contribuendo elleno a dare veruna idea che conduca alla formazione del bilancio"32 e lo stesso avveniva anche ora, benché la prospettata abolizione delle giurisdizioni interne rendesse i movimenti commerciali interprovinciali per lo meno decisivi tanto quelli interstatali. La questione restava comunque in sospeso e lo si sarebbe visto più avanti nel tempo.

Del resto, quando sul finire di novembre del 1773 Verri, una volta messo a punto il progetto cui era stato delegato, aveva iniziato a discuterne il testo insieme a un ristretto gruppo di altri consiglieri del Magistrato Camerale, non aveva registrato riserve al riguardo. Si trovava allora per altro in buona compagnia perché ad affiancarlo era innanzitutto uno come Cesare Beccaria, che già durante il biennio della sua docenza in Scienze Camerali alle Scuole Palatine, si era pronunciato nettamente contro la sopravvivenza delle barriere doganali interne. ${ }^{33} \mathrm{E}$ non mostrava certo ora di volersi ricredere.

È vero: non erano più i tempi di quando sulle pagine de "Il Caffè" si cimentava in una esercitazione a sfondo economico-matematico sui contrabbandi, alla ricerca di una manovra daziaria "ottimale" per contrastarli. ${ }^{34} \mathrm{E}$ inoltre la sua carriera di pubblico funzionario da poco iniziata si stava orientando principalmente ormai verso "la provincia d'annona" pur da posizioni sempre meno assimilabili a quelle ben più radicali sostenute in materia da Verri e tuttavia ugualmente ispirate da un medesimo orientamento a favore della libertà di commercio dei grani. ${ }^{35}$ Ma sul piano della riforma daziaria, la convergenza di posizioni aveva

32 G. Tonelli, Commercio di transito e dazi di confine nello Stato di Milano tra Sei e Settecento, in A. Torre, Per vie di terra. Movimenti di uomini e di cose nelle società di antico regime, Milano, F. Angeli, 2007, p. 98.

33 G. Bognetti, Cesare Beccaria, in V. Negri Zamagni, P.L. Porta, Dizionario enciclopedico del pensiero economico in Italia, Roma, Istituto dell'Enciclopedia Italiana, in preparazione.

34 Cfr. C. Beccaria, Tentativo analitico su $i$ contrabbandi, in G. Francioni, S. Romagnoli (a cura di), «Il Caffè» 1764-1766, Torino, Bollati Boringhieri, 1993, pp. 173. 175, con M. Bianchini, Cesare Beccaria e Pietro Verri: il metodo geometrico, il reale e l'immaginario, in «Il pensiero economico italiano», a. IV (1996), n. 2, p. 24.

35 Cfr. C. Capra, I progressi della ragione. Vita di Pietro Verri, cit., pp. 292-303, con A. Carera, Cesare Beccaria magistrato in «provincia d'Annona», cit., pp. 425-446. 
avuto modo ugualmente di manifestarsi, in forza del disegno di unificazione doganale interna su cui poggiava, ma non meno della comune visione circa il riequilibrio spontaneo dei rapporti di scambio con l'estero che l'ispirava, mutuata da Hume. ${ }^{36}$

Ad alimentare una comune visione in materia, era anche il modo con cui concepivano entrambi, come è stato opportunamente messo in rilievo, la politica commerciale, facendola diventare strumento di "massimizzazione dell'occupazione", attraverso aggravi daziari sulle esportazioni di materie prime e sulle importazioni di manufatti. ${ }^{37}$ Sembrava quasi che almeno sotto questo profilo, si fosse ristabilita tra i due quella comunanza di intenti che li aveva animati ai tempi della Accademia dei Pugni. La quale pareva ora potersi estendere anche a un altro esponente di quel sodalizio, vale a dire il Secco Comneno, ${ }^{38}$ partecipe pure lui dell'opera di consultazione avviata dal Verri. E dopo un primo appuntamento tenutosi il 22 novembre del 1773, era venuto anche il momento di contarsi. Un mese dopo, intervenendo a un'altra seduta fissata per il 24 dicembre, l'ex fermiere Mellerio, aveva rivolto serie critiche al progetto in discussione. ${ }^{39}$ Suo punto di attacco era stato il riproporre un quesito mai sopito e per il quale bisognava tornare a chiedersi "se conveniva tener divise le giurisdizioni dello Stato, ovvero considerare tutto il Milanese in un corpo solo" Questa volta a imporlo era, secondo lui, il "punto della giustizia", in quanto non poteva ritenersi "giusto far portare a una Provincia que' pesi che si sottraggono all'altra". Al che Verri e di conserva anche Beccaria e Secco Comneno ("noi tre" appunto), non avevano avuto difficoltà a replicargli che così non era perché "ogni Provincia avrà soglievo sopra alcuni capi e aggravio sopra altri", e che questo valeva anche per il Ducato, benché si ritenga "favorirsi col nuovo sistema" e proprio a cominciare da Milano. Il meccanismo della "perequazione" adesso invocato aveva del resto già mostrato di funzionare, allorché sul fronte "del prediale" si era riusciti a far prevalere i "principi superiori del ben pubblico" contro "l'originario

36 Cfr. A. Moioli, Nota introduttiva, cit., p. 6, con G. Bognetti, Cesare Beccaria, cit.

37 Idem, Governo dell'economia e teoria della politica economica. Testo della relazione presentata al Convegno, infra.

38 Su Pier Francesco Secco Comneno, ved. C. Capra, I progressi della ragione, cit., p. 187.

39 "Riflessioni preliminari per la formazione d'una nuova Tariffa", in Archivio Verri, c. 434 , fasc. 3. 
antichissimo uso di ripartire il carico" che non era certo dei più efficienti ed equilibrati. Lo stesso ora poteva ripetersi "senza ingiustizia" e sempre con la stessa logica perequativa sul fronte doganale, in forza di "un tributo uniforme, intellegibile e lontano dagli inviluppi attuali". ${ }^{40}$

Mellerio aveva comunque in serbo un altro motivo di critica più insidioso che riguardava l'inevitabile rischio di una contrazione del gettito per effetto degli aggravi introdotti con la tariffa unica, sia perché incentivanti come non mai il contrabbando, sia a motivo delle diminuite importazioni che una domanda in flessione avrebbe recato con sé. Smentirlo da questo punto di vista era piuttosto difficile in assenza, anche nel bilancio per il 1769, di una valutazione del cosiddetto "prodotto dei daziati". Verri si limitava a parlare in proposito di "dati incalcolabili". Non era proprio così e tuttavia, in mancanza di adeguati riscontri al riguardo, non gli rimaneva che far notare al suo interlocutore come la via dei ritocchi da lui proposta in alternativa, non era per nulla una svolta: "non sarebbe che stampare la Tariffa vegliante". ${ }^{41}$

A quel punto, non era rimasto che ribadire ciascuno le proprie posizioni e Verri, nel prenderne atto, annotava: "Ci separammo noi tre persuasi col primo sentimento e il sig. Consigliere Mellerio col suo". Confessava però subito dopo che forse "sia più prudente a lasciar le cose come sono e fare come il Cons.re Mellerio propone". Se non che bisognava anche adeguarsi alla volontà della Corte e questa non avrebbe certo gradito un simile ripensamento perché poteva "sembrare una voglia di travagliare meno e mancanza di energia per fare qualche utile e vasta riforma". ${ }^{42}$ Era quindi meglio non debordare dai propositi iniziali e così, dopo un'altra consultazione compiuta il 4 febbraio 1774, il progetto era stato presentato ufficialmente al Magistrato Camerale il 30 maggio dello stesso anno e approvato il 6 luglio seguente.

Era stata questa una decisione unanime, presa sulla base di un testo che nel suo articolato sviluppo riproduceva "il disegno e l'idea" del nuovo sistema daziario, nell'ipotesi di una sua compiuta rifusione, dalla quale far scaturire l'auspicato regime tariffario unificato. Il "partito" intorno al quale Verri si andava spendendo non poteva del resto che essere questo, sapendolo per giunta pienamente condiviso, come si è visto,

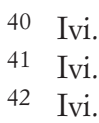


dal Beccaria e dal Secco Comneno ai quali non a caso andava un esplicito riconoscimento nella introduzione del suo rapporto: per aver avuto "la compiacenza più volte di unirsi meco a trattare di queste viste". ${ }^{43}$ Ma poi sempre lui doveva ammettere che operando secondo le "massime" con loro concordate, si sarebbe dovuto affrontare un percorso attuativo piuttosto accidentato (almeno in una fase iniziale), non esente tra l'altro da rischi di scarsa efficacia impositiva. Ecco perché egli tornava a fare propria una riflessione già da lui annotata ai margini del già ricordato confronto avuto con il Mellerio e secondo la quale sarebbe stato ragionevole anche seguire "il partito di ritoccare parzialmente la Tariffa e ristamparla", ciò comportando "minore rivoluzione, minori querele e nessun pericolo per l'erario". Nel qual caso si imponeva però una scelta di campo che egli rinviava al "supremo arbitrio" della Corte. ${ }^{44}$

Per il consigliere Pellegrini invece questa alternativa nell'una o nell'altra direzione non si poneva nemmeno. Scrivendone di lì a poco in un promemoria inoltrato a Vienna, dichiarava a chiare lettere che il progetto verriano di tariffa unica, quello stesso che lui aveva appena sottoscritto, andava accantonato proprio perché la sua adozione si prestava a numerose e serie controindicazioni. A cominciare dagli ammanchi procurati alle casse pubbliche dal venir meno delle gabelle interne e dal manifestarsi di un contrabbando che si sarebbe reso più aggressivo. ${ }^{45}$ Ben sapeva così di essere pienamente convincente. Nelle sedi governative della Capitale asburgica prospettare una simile eventualità stava diventando un argomento di sicuro successo, in forza del quale si poteva anche ammettere che la modernizzazione degli assetti doganali fosse un obiettivo di per sé rinviabile. Era del resto per questa stessa ragione che era stata abbandonata sul nascere un'analoga proposta di riforma avanzata proprio allora dal capo del dipartimento delle dogane Coblenz con riferimento alla parte cisleitanica della Monarchia. ${ }^{46}$

I tempi per l'unificazione doganale dello Stato milanese si erano

43 P. Verri, Progetto d'una tariffa della Mercanzia per lo Stato di Milano, del 30 maggio 1774, in appendice a A. Moioli, La questione della riforma daziaria nello Stato di Milano, cit., p. 890.

44 Idem, p. 895.

45 Ivi, p. 887.

46 F.A.J. Szabo, Kaunitz and enlightened absolutism, 1753-1780, Cambridge, Cambridge University Press, 1994, p. 888. 
così lungamente dilazionati e "l'arabesco pasticcio" del tariffario preesistente aveva trovato modo di mantenersi in molta parte della sua conformazione originaria. $\mathrm{Al}$ massimo si era ottenuto che intervenendo su di essa, si allargassero le maglie della tassazione applicata alle dogane interne e si innescasse per queste ultime un processo di erosione in grado di prefigurare gli esiti agevolanti dal punto di vista della commercializzazione, connessi con la loro contrazione o esenzione. Era quanto peraltro si era già fatto rilevare durante i contatti preliminari che avevano accompagnato la progettazione del regime daziario unificato. Ma allora ad essere chiamata in causa era l'uniformazione impositiva introdotta nel 1765 per la circolazione infrastatale delle sete, dei formaggi e del lino, a sostegno della valenza strategica insita nella ristrutturazione tariffaria progettata. ${ }^{47}$

Ben si sapeva ormai che non era certo questo il percorso che si doveva seguire per metterla in pratica. Verri, è vero, se ne stava ormai fuori, ma la sua estraneità non significava che la riforma fosse per lui da considerarsi fallita. In realtà si stava verificando esattamente l'opposto e proprio a partire dalla sede istituzionale che l'aveva visto protagonista. Adesso a guidare la pur lenta marcia di avvicinamento alla tariffa unica non era più infatti il Magistrato Camerale, quanto invece un nuovo organismo come la Camera dei Conti, chiamata sul finire degli anni '70 a mettere in atto quegli adempimenti tecnici che dovevano finalmente condurre alla definizione in dettaglio della relativa nuova griglia daziaria, imposta se non più ovviamente sul fronte degli scambi interni (perché resi esenti), su quelli dell'import-export e dei transiti. Non si poteva però per questo prescindere dagli elaborati di Verri e in special modo da quello predisposto per il 1769, che attendeva ancora di essere valorizzato e che da Vienna veniva additato come un necessario punto di partenza da comparare e integrare con un altro, questa volta da costruire ex novo, per il 1778. Lo stesso avrebbe dovuto però diventare un bilancio "di Commercio e di Finanza", reso tale dal fatto di dare uno specifico spazio ai gettiti conseguiti in corrispondenza rispettivamente degli scambi con l'estero, del commercio di transito e della circolazione interna, per la prima volta così rilevata, seppure soltanto nella sua entità fisica. ${ }^{48} \mathrm{Si}$ col-

47 "Riflessioni preliminari per la formazione d'una nuova tariffa", cit.

48 G. Tonelli, Baldassarre Scorza e la riforma daziaria nella Lombardia asburgica, in «Nuova economia e storia”, Pisa, a. I (1997), p. 44. 
mavano così due lacune presenti nei conti verriani: l'una già accennata che riguardava le cifre dei così detti "daziati"; l'altra derivante dalla mancata misurazione dell'impatto che avevano le correnti di traffico tra le giurisdizioni doganali e quelle del transito sul complesso degli scambi con l'estero. Il volume dei traffici in entrata e in uscita era poi posto a confronto con quello risultante per il 1769 , da tradursi in valore sia a prezzi costanti rispetto a tale anno, sia a prezzi correnti calcolati in media sul decennio 1772-1782.49

Allargare lo spettro delle basi di calcolo su cui fondare la struttura del nuovo bilancio era tutt'altro che un atto dovuto in senso formale. Era invece una condizione imprescindibile a cui affidarsi per definire finalmente in dettaglio la tariffa riformata, altrimenti soltanto teorizzata o al più ridotta a uno stato di pura esemplificazione, com'era infatti successo di dover fare allo stesso Verri. Ben lo si sapeva del resto presso la Camera dei Conti che così operando non sarebbe più stato come prima e di ciò era a maggior ragione consapevole chi, in qualità di suo segretario, si era trovato tra il 1780 e il 1782 a impostare e a realizzare un elaborato a ragione di recente giudicato "quanto di più pregevole l'amministrazione lombarda abbia prodotto in questo campo nel secolo XVIII" ${ }^{50}$ Si trattava di Baldassarre Scorza, un funzionario che aveva operato alle dipendenze della Ferma e che professionalmente era cresciuto all'ombra del Greppi sino a diventare un diretto collaboratore anche del Verri ai tempi dell'appalto in cointeressenza del "Dato della Mercanzia” e dell'amministrazione interinale che ne era seguita. Ed era stato proprio costui a manifestare nei suoi confronti uno speciale apprezzamento per le doti personali e le eccellenti competenze tecniche in campo mercantile e finanziario, facendosene interprete presso i dicasteri viennesi competenti. ${ }^{51}$

Non si era certo sbagliato e ne aveva la conferma proprio ora che questo funzionario di medio calibro si era posto a costruire un bilancio risultato da subito così ben riuscito da assumere un valore esemplare, paragonabile se non superiore a quello attribuibile alle analoghe rileva-

49 Ivi, p. 51.

50 C. Capra, Il Settecento, in D. Sella, C. Capra, Il Ducato di Milano dal 1535 al 1796, Torino, Utet, 1984 , p. 473.

51 G. Tonelli, Baldassarre Scorza e la riforma daziaria nella Lombardia asburgica, cit., p. 37. 
zioni di cui Verri era stato maestro. Evidentemente il bagaglio tecnico di cui questi si era reso portatore era ormai entrato nella prassi amministrativa del governo milanese a tal punto da consentire a un operatore di profilo intermedio come lo Scorza di metterlo in pratica con particolare successo. Non era certo frequentatore dell'economia matematica come invece aveva mostrato di essere l'animatore della "Scuola di Milano", giunto tra l'altro, proprio in vista del suo elevato bagaglio teorico, a polemizzare persino con il Carli circa la validità scientifica dei due bilanci da lui fatti compilare per il biennio 1766/67, quand'era ancora presidente del Supremo Consiglio dell'Economia..$^{52} \mathrm{Ma}$ il suo limitato bagaglio culturale che pure traspariva dietro le numerose citazioni con cui aveva infiorato il commento finale del proprio elaborato, ${ }^{53}$ non gli aveva impedito di rivelarsi un abile interprete dell'analisi di statistica comparata avente come obbligato punto di riferimento, per volontà dello stesso governo, il conto verriano riferito al 1769 .

L'illuminismo delle riforme civili poteva anche condurre, se non altro per via di contaminazione, a simili esiti. Ma non solo a questo. Perché, una volta completati i cinque tomi del nuovo conto, a questo ispettore di fresca nomina della Camera dei Conti era toccato di collaborare con la stessa nella stesura dei primi abbozzi tariffari di quello che sarebbe diventato il nuovo regime daziario unificato. Nonostante le pressioni venutegli da Vienna e le previsioni circa l'attivismo che il know how conseguito con il suo bilancio gli avrebbe consentito di esprimere, egli non era però riuscito a combinare molto. Non si poteva certo chiedere a lui di svincolarsi dalle tensioni insorte tra il suo dicastero e le autorità di Vienna intorno alle "massime" riformatrici formulate da Verri. In effetti non era più in gioco soltanto l'uniformazione delle dogane associata alla soppressione delle giurisdizioni interne, ma anche l'impronta interventista che la stessa avrebbe dovuto assumere a sostegno delle produzioni manifatturiere interne.

Si era creata così una situazione di stallo che non era stata scossa nemmeno quando nel 1784 era stata emanata la patente per l'abo-

52 A. Moioli, Nota introduttiva a P. Verri, Bilanci del commercio dello Stato di Milano, in Edizione Nazionale delle Opere di Pietro Verri, vol. II, cit., pp. 479-481.

53 C.A. Vianello, Discorsi inediti di Baldassarre Scorza sui bilanci dello Stato di Milano del 1769 e del 1778 e sui porti di Trieste e di Nizza, Milano, 1938, pp. 84, 100, 140,165 . 
lizione dei dazi interni nelle provincie austro-boeme..$^{54}$ Lo Scorza l'aveva subita, ma guardandosi bene dall'identificarsi nelle posizioni che la Camera era venuta sostenendo a favore delle esportazioni che l'agricoltura lombarda alimentava e contro quindi le misure protezioniste volte a contrastare le importazioni di manufatti. E quando ai primi del 1786, per disposizione di Kaunitz, nella gestione della materia tariffaria era subentrata l'Intendenza Generale, egli si era trovato di nuovo in prima linea ad affiancare il Lottinger, preposto a tale ufficio, che ora, rompendo gli indugi, era incaricato di chiudere il più in fretta possibile la questione. L'operazione di rifacimento della tariffa venne terminata così entro l'anno e questa entrava in vigore con il $1^{\circ}$ gennaio 1787 . Fu però il frutto di un compromesso perché la soppressione delle gabelle sulla circolazione interna si accompagnò all'adozione generalizzata dei dazi di confine vigenti nell'ex giurisdizione della capitale ambrosiana che dal lato delle importazioni si prospettavano come i più onerosi. Di modo che si era bensì ottenuto di far crescere anche sensibilmente gli introiti doganali, ma senza che vi si accompagnasse, come invece aveva previsto Verri, un effettivo slancio impresso all'interscambio. ${ }^{55}$

Come del resto poteva essere diversamente di fronte a una situazione in cui i prevalenti rapporti commerciali con gli Stati limitrofi venivano ora regolati da dazi unici sostitutivi dei diritti analoghi in precedenza esatti in misura differenziata, prendendo come base di riferimento il tariffario della giurisdizione di per sé la più tassata? Non era certamente questo il metodo impositivo sollecitato dalle massime verriane per la riforma. Ma andava pur sempre bene a Vienna che, una volta soddisfatte le ragioni del pubblico erario, non aveva di mira altro che l'obiettivo di contenere le importazioni dei manufatti di più largo smercio e a tutto vantaggio delle produzioni interne, ma senza penalizzare allo stesso tempo l'afflusso dei beni di lusso che continuavano ad alimentare un proficuo commercio di intermediazione facente capo a Milano. Si può allora capire perché mai da un confronto tra le bilance commerciali per il 1769 e il 1778 con quella abbozzata per il 1790 dal Piccinini (il nuovo capo dell'ufficio di revisione della Camera dei Conti succeduto allo Scorza), emerga l'evidenza di come i dazi riformati

54 C. Capra, Il Settecento, cit., p. 456.

55 A. Moioli, La questione della riforma daziaria nello Stato di Milano, cit., p. 888. 
abbiano ostacolato più i flussi in entrata di molteplici articoli destinati al maggior consumo che non quelli dei beni di lusso. ${ }^{56}$

Ciò darebbe allora ragione delle contrastanti reazioni che hanno accompagnato e seguito l'avvento di una tariffa così concepita e sull'onda delle quali ha preso ben presto avvio il tentativo di modificarla. Nel 1791 infatti, a soli cinque anni dalla sua entrata in vigore, veniva istituita una Delegazione Daziaria incaricata, sulla base delle risultanze ancora ritenute valide del bilancio "di finanza e commercio" redatto dallo Scorza, di procedere a una revisione della struttura impositiva in corso per i settori merceologici più importanti. ${ }^{57}$ Dei suoi lavori però è rimasta soltanto una ricca documentazione e niente di più. Le sue proposte, forse a motivo del loro orientarsi in senso troppo marcatamente protezionista e quindi in contrasto con gli interessi di indole agricolocommerciale prevalenti nell'ambiente, sono rimaste lettera morta.

56 Idem, L'economia lombarda verso la maturità dell'equilibrio agricolo-mercantile, in AA.VV., Cesare Beccaria tra Milano e l'Europa, cit., p. 347.

57 Idem, Note sulla struttura del commercio estero dello Stato di Milano nella seconda metà del Settecento, in AA.VV., Studi in onore di Gino Barbieri, Verona, Spem, 1983, vol. II pp. 1052 passim. 